\title{
Madrid pide pan
}

\section{Madrid asks for bread}

(iD) Irene Valiente Guerrero ${ }^{\square}$

Madrid, España

\begin{tabular}{llll}
\hline Recibido: 25/08/2020 & Revisado: 02/09/2020 & Aceptado: 04/09/2020 & Publicado: 25/10/2020 \\
\hline
\end{tabular}

\section{RESUMEN}

Desde que se declara el estado de alarma en Madrid, se ha podido observar cómo el centro de España ha sido sacudido por una crisis social sin precedentes, que se va incrementando según pasan los meses. Las instituciones no han hecho lo suficiente para que las llamadas "colas del hambre" no se repitiesen por todos los rincones de Madrid. Esto ha sido la viva imagen de una ciudad que sin el desarrollo de las redes vecinales y despensas solidarias no hubiese sobrevivido.

Palabras clave: Madrid, solidaridad, asociaciones vecinales, despensas solidarias.

\section{ABSTRACT}

Since the state of alarm was declared in Madrid, it has been possible to observe how the central part of Spain has been shaken by an unprecedented social crisis, which is increasing as the months are passing. Institutions have not done enough to avoid the so-called "hunger queues", lines of people in front of residents' associations waiting for food provided by the neighbours to survive. This has been the spitting image of a city that without the development of such networks and solidarity pantries would not have survived.

Keywords: Madrid, solidarity, residents' association, solidarity pantries. 


\section{INTRODUCCIÓN}

Una semana previa a la declaración del Estado de Alarma en la Comunidad Autónoma de Madrid, las asociaciones vecinales decidieron, por prevención, echar el cierre a sus instalaciones y a todas las actividades que estaban programadas para ese mes. El cierre fue con la idea de que en veinte días volvería a la normalidad, nadie imaginó que esa situación se iba a alargar casi tres meses. La situación de crisis en las familias y en los barrios de Madrid se dejó ver apenas unas semanas después, cuando las asociaciones vecinales y colectivos sociales recibían peticiones de ayuda de sus vecinos y vecinas, por diversas situaciones que impedían a las familias hacer la compra, ir al centro de salud o recoger sus medicamentos en las farmacias. En ese momento algunas asociaciones y colectivos decidieron ayudar a aquellas personas que eran de riesgo a hacer las compras. Este proyecto se nutrió de voluntarios/as de los propios barrios que salían a hacer la compra a sus vecinos/as, acercar medicamentos de las farmacias o pruebas de los centros de salud (que en ese momento permanecían cerrados). Fue algo sorprendente que sin una coordinación previa de todos estos colectivos se actuara de la misma manera.
Fue entonces cuando se creó el grupo de WhatsApp para la coordinación de acciones conjuntas de las distintas "redes de solidaridad" de toda la Comunidad de Madrid. En ese momento se entendió que se debía dar un paso más, señalar y responsabilizar a las instituciones (Ayuntamiento de Madrid y Comunidad de Madrid) para que se adelantara a una situación de crisis y emergencia social que todos los actores sociales veían que se acercaba.

\section{DESARROLLO}

Lo primero que se hizo fue sacar un comunicado criticando las palabras de la Vicealcaldesa Begoña Villacís. Esta señalaba que "Si tenemos que sacar algo bonito de esto, es que nos ha hecho descubrir que tenemos vecinos, compartimos mucho más, nos sentimos más madrileños que nunca" (Fuente: Periódico "El País" 20 de marzo). Desde estas entidades se entendió que se estaba intentando sacar rédito político, más bien partidario, de una emergencia social que golpeaba a los vecinos/as de una manera más severa de lo que lo fue en el 2008. En este comunicado comenzó una acción por redes sociales con el Hashtag \#SeLavanLaCaraConNuestrasManos (Figura 1).

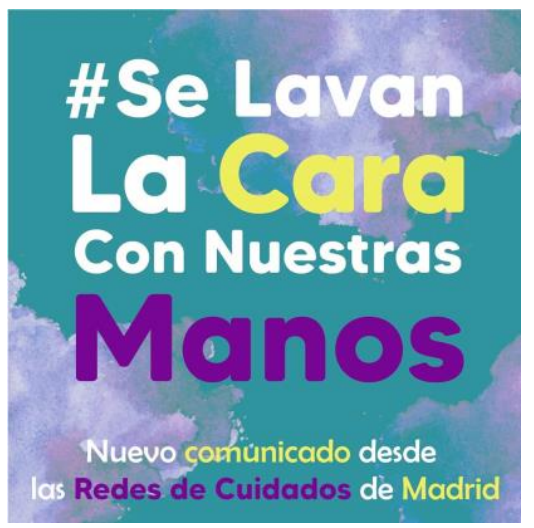

Figura 1. Primera campaña de las Redes de Cuidados de Madrid 
Gracias a estas acciones por redes sociales, los mass media comenzaron a hacerse eco de estas acciones vecinales, que no eran espontáneas, sino que se organizaron gracias a las asociaciones y colectivos vecinales de los barrios y pueblos de Madrid. Por eso la acción tenía como objetivo resaltar que el Ayuntamiento de Madrid se estaba aprovechando del trabajo voluntario y solidario de las redes vecinales, cuando tiempo atrás, los tres partidos políticos que gobiernan en el Ayuntamiento y en la Comunidad (Partido Popular y Ciudadanos con el apoyo de Vox) habían basado su campaña política y propuestas en desmantelar todo el tejido asociativo y cualquier lucha vecinal, calificando a las asociaciones como "chiringuitos" del sector más progresista de los anteriores gobiernos.

En este momento se entendió que la batalla de las asociaciones y colectivos vecinales iba a ser dura, nunca se pudieron imaginar cómo iba a evolucionar esta situación.

Después de algunas semanas (mediados del mes de abril), más de sesenta entidades comenzaban a abrir las puertas de sus locales para la entrega de comida a familias que por diversas situaciones no podían hacer frente al pago de la compra, lo que suponía muchas familias en una situación de pobreza sin precedentes en la Ciudad de Madrid.

La mayoría de estas redes vecinales fueron evolucionando a "Despensas Solidarias", encargadas de recoger y repartir comida a las familias que se encontraban en situaciones muy precarias, sin trabajo, sin cobrar los subsidios y con menores a su cargo a los que alimentar.

Se generó una red de voluntarios/as, alrededor de esas asociaciones y colectivos, para comenzar a repartir comida. Fue entonces cuando estos actores funcionaban a la par, con los mismos fallos, las mismas dudas y las mismas peticiones a las administraciones, fue cuando la coordinación tomó forma y se creó "Redes de Cuidado de Madrid"1, con una participación de más de 60 entidades (Figura 2). En todos los

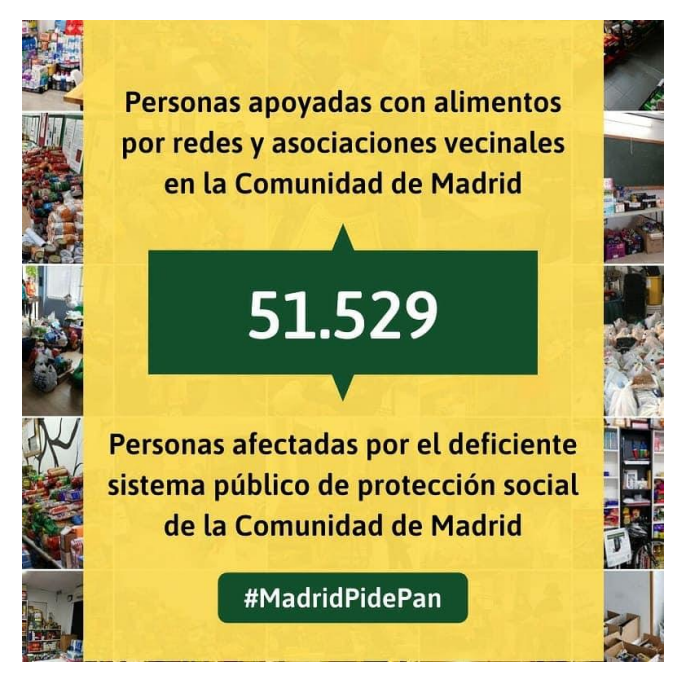

Figura 2. Infografía FRAVM 
barrios la demanda de comida comenzó a incrementarse, dejando ver que la capacidad de estas despensas tenía límite y un fin, ya que se estaba repartiendo comida en los locales principalmente de asociaciones vecinales y con un matiz de emergencia social.

No todos los barrios lo sufrieron igual, fue entonces cuando las llamadas "colas del hambre" se hicieron eco de manera estatal y europea, gracias a los medios de comunicación que hicieron un gran seguimiento y sirvieron de altavoz para todas estas entidades.

El Gobierno de España sacó una medida en el mes de marzo para ayudar a las empresas a tener cerrado y poder pagar a sus trabajadores, Ios ERTE (Expediente de Regulación Temporal de Empleo), el gobierno aseguraba el 75\% del sueldo de los trabajadores. El problema vino cuando a la mayor parte de estos trabajadores no se les pagó el ERTE hasta el mes de mayo o junio, lo que hizo aumentar estas "colas del hambre".

También hay que resaltar que durante estos meses las ONGs, Cáritas, Cruz Roja y el Banco de Alimentos de Madrid se encontraban sobrepasados y con pocos productos que dar en sus cestas, lo que supuso un incremento de las familias en las Despensas, ya que de alguna forma se sustituyó su trabajo por las entidades locales. Esto supuso que muchas entidades y colectivos asumieran una actitud crítica ante las entidades que históricamente se han encargado de la caridad y de la ayuda humanitaria en los momentos de las crisis.

En el mes de mayo la situación política era diferente, se repetían las concentraciones y manifestaciones en las puertas de Ayuntamientos, Juntas de Distrito (son los órganos de gobierno en la Ciudad de Madrid que está dividida en 21 distritos), ya que la situación se agravaba y las administraciones no hacían lo necesario para paliar, ayudar y sostener la ayuda de alimentos a las familias que lo necesitaban. Hablamos de una ciudad que en cifras del año 2019 encontramos una diferencia entre estos distros de la renta per cápita media reseñable. ${ }^{2}$ Las únicas ayudas que dieron desde las Juntas y la Comunidad eran las relacionadas con las familias cuyos hijos e hijas recibían la beca de comedor, algo que fue muy criticado tras las palabras de la Presidenta de la Comunidad de Madrid Isabel Diaz Ayuso, en la que reconocía que los menús infantiles que se llevaban repartiendo durante este periodo se servían por parte de Telepizza y Rodilla y que la comida que estos repartían no era nada adecuada para los menores, no era saludable y no se modificó hasta tres meses después de la declaración del Estado de Alarma. El ayuntamiento por su parte no contrató a más personal para la primera asistencia en Servicios Sociales, los teléfonos estaban colapsados y era imposible que atendieran a esa cantidad de familias, las redes y despensas solidarias pasaban los datos a servicios sociales siempre que las familias estuviesen de acuerdo, que en un noventa por ciento de los casos así era. El dinero sobrante de los contratos de las fiestas de los barrios sí se destinó para comidas a domicilio para las familias registradas en Servicios Sociales, una comida insuficiente y preparada, que impedía la independencia de las propias familias y la incapacidad de poder desayunar o merendar, ya que estos menús sólo se entregaban para la comida y la cena. La crispación política e ideológica se podía notar en las calles de Madrid, algunos colectivos fascistas (como Hogar Social Madrid) repartían alimentos solo a personas españolas, aprovechándose de su situación de precariedad máxima para el reparto de su propaganda política.

${ }^{2 h t t p s: / / w w w . e x p a n s i o n . c o m / e c o n o m i a / 2019 / 09 / 12 / 5 d 7 a 1 c 78 e 5 f d e a 4 b 218 b 458 e . h t m l ~}$ 
Al llegar el mes de agosto muchas despensas llegaron a su fin, muchas de ellas llevaban más de tres meses repartiendo comida, con el desgaste que supone eso, el número de familias que se iba incrementando, estas despensas fueron cerrando, quedando a día de hoy algunas abiertas, pero con muchas limitaciones. Algunas de estas asociaciones y colectivos analizaban que la situación de emergencia ya había pasado y que las instituciones habían tenido tiempo de sobra para dar una respuesta a estas familias, con el pesar de muchas de ellas, estas despensas han ido cerrando en todo Madrid.

No es hasta finales del mes de agosto cuando el Ayuntamiento de Madrid decide sacar la tarjeta monedero y poder ayudar así a las familias más desfavorecidas de la ciudad. Esta medida se llevaba pidiendo años atrás por parte de todas estas entidades (Figura 3).

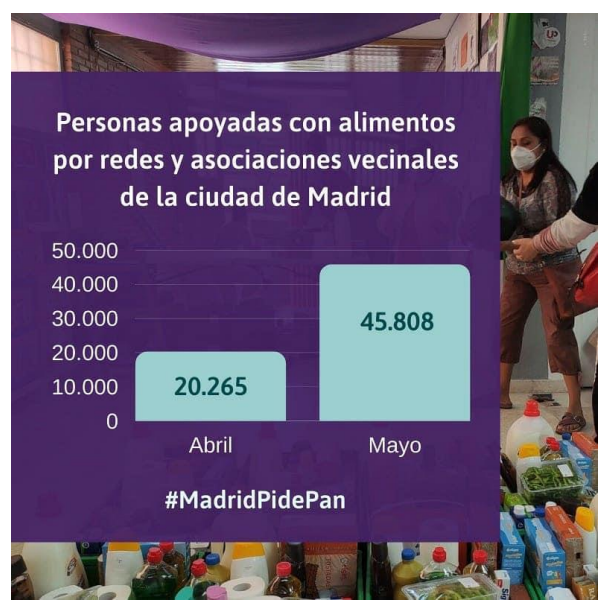

Figura 3. Infografía FRAVM

La realidad a día de hoy es que la situación de las familias de Madrid no ha mejorado, el hambre sigue siendo parte del día a día de muchos menores, el paro y la precariedad golpea a los barrios y pueblos de Madrid, mientras que los responsables políticos de las instituciones miran para otro lado, haciendo caso omiso de las peticiones de las entidades y colectivos que han tenido que ver cómo sus vecinos y vecinas han tenido que hacer colas para recibir alimentos.

\section{Perfil de las familias y voluntarios}

Para poder entender la situación de estos últimos meses en Madrid, es importante saber qué tipo de familias asistían a la recogida de alimentos y de qué tipo de voluntarios/as se nutrían estas despensas solidarias, para poder entender por qué se ha llegado a esta situación.

Las familias que principalmente contactaba con las Despensas eran familias compuestas por personas migrantes, personas que trabajan en la economía sumergida (cuidadoras de ancianos, limpiadoras domésticas, cuidadoras de menores, personas sin contrato) y que por tanto no podían recibir ningún subsidio; personas sin permiso de residencia en España, personas pendientes de asilo político, personas que aún no habían cobrado el ERTE, familias muy numerosas con los dos progenitores 
desempleados, familias monomarentales o personas con ningún arraigo familiar en Madrid.

De todos estos casos, el rostro de todas estas personas, ha sido el de las mujeres, las mujeres como las proveedoras de alimentos y de sostener a la familia, mientras que los hombres, desempleados, asumen el papel de la vergüenza. Como siempre el rostro de la pobreza pertenece a la mujer y en esta ocasión se ha vuelto a repetir. Las mujeres, madres, esposas, tías, abuelas o hijas, han sido las valientes que se han atrevido a dar rostro a esta crisis social.

Muchas de estas familias se incluían como voluntarios/as de la propia despensa de alimentos (Figura 4), la idea de estas despensas era generar redes de apoyo mutuo, la solidaridad como pilar ideológico de estas despensas, alejado de la caridad cristiana o religiosa que impera cuando se habla de la ayuda humanitaria.

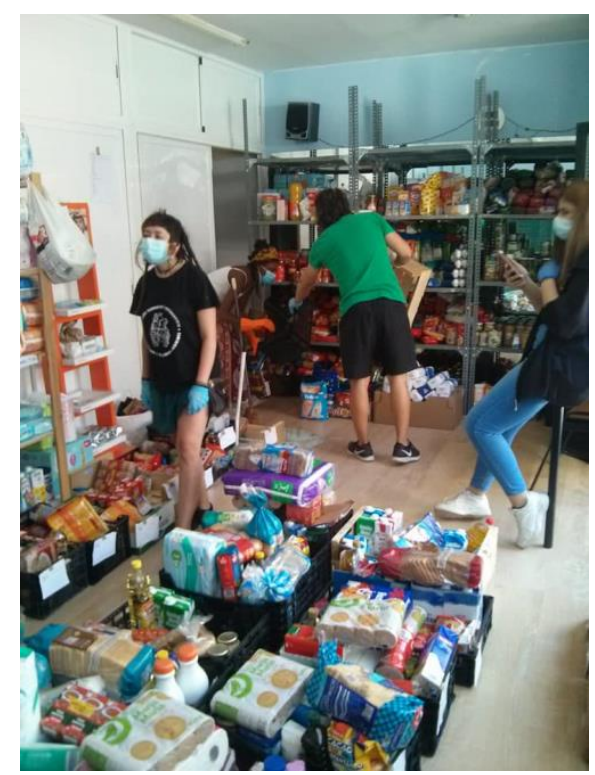

Figura 4. Despensa Solidaria La Elipa

Las Despensas no hubiesen funcionado sin los voluntarios/as, estas personas eran vecinos/as de los propios barrios, personas que se encontraban teletrabajando, en ERTE o desempleadas, cercanas a las asociaciones, vecinos/as que siempre han querido ayudar y que comparten los principios de la colectividad vecinal.

El problema vino cuando estos voluntarios/ as volvieron a sus respectivos trabajos, se fueron de vacaciones, un factor importante a la hora de tomar la decisión del cierre de las despensas. Sin una cantidad importante de personas voluntarias no era posible asumir el reparto de alimentos, la logística y la preparación de esta ayuda.

Existía otro tipo de voluntariado menos activo pero fundamental para la red vecinal que sostenía esto, aquellas personas que donaban todas las semanas un carro de la compra, dinero en metálico o comercios locales que situaban cajas para que sus clientes donasen algo de su propio comercio, todo para poder comprar productos específicos (cosas para 
bebés, higiene, productos para personas con alergias o intolerancias, productos frescos, etc.) Estas personas fueron fundamentales, pero también fueron disminuyendo, por tanto, las despensas se encontraron sin voluntarios activos ni pasivos, sin donantes.

El perfil es importante a la hora de analizar las personas que componen los barrios de Madrid y las propias desigualdades que se dan entre los sectores más precarios de la sociedad. Los barrios con menos renta per cápita de Madrid fueron los más activos y con despensas con más capacidad (hablamos de Vallecas, Carabanchel, Latina o Usera), esto demuestra que hay solidaridad de clase y empatía. Gracias a estas dos características las familias en Madrid han podido llevarse un plato caliente a la boca todos los días.

\section{Redes digitales y brecha digital}

La importancia de las redes sociales en esta pandemia ha sido fundamental para dar a conocer las iniciativas vecinales, tanto las redes de solidaridad de los primeros meses, como las despensas solidarias. La capacidad de poder llegar a muchas personas de manera online ha facilitado la organización de estas acciones. El uso de las herramientas telemáticas para poder mantener reuniones, han servido para continuar con el trabajo diario de estas asociaciones y colectivos.

Pero esto acarrea dos consecuencias fundamentales, derivadas de la brecha digital. Los dos actores que más han salido perjudicados en esta época han sido las personas mayores y las familias con condiciones materiales de vida precarias (como las familias usuarias de las despensas solidarias). El acceso a las nuevas tecnologías se hace complicado para las personas mayores que no tienen un uso cotidiano con un ordenador o con un smartphone, esto supuso que las personas que contactaron con las redes solidarias eran sus familiares o personas cercanas, lo que dificultó la comunicación con algunas personas y por lo que muchas de ellas no pudieron acceder a estas ayudas.

Las familias usuarias de las despensas, muchas de ellas, no tienen acceso a internet, lo que supone que muchos menores no han podido continuar las clases online (produciendo una brecha en el nivel educativo público) y otro aspecto importante a señalar, es que las propias familias no podían acceder a la petición de ayudas públicas, como el IMV (Ingreso Mínimo Vital), ayuda que lanza el Gobierno de España para ayudar a estas familias. Esta ayuda solo se podía tramitar de manera online, por lo que muchas despensas solidarias habilitaron salas con ordenadores y voluntarios/as con conocimientos en internet, para ayudar a estas familias a poder pedir esta ayuda.

La brecha digital es un problema a día de hoy en la sociedad española, ya que mucha gente se queda fuera de la vida estudiantil y laboral por no tener un acceso gratuito a Internet o a los medios tecnológicos.

Propuestas y respuestas políticas por parte de los actores implicados

El portavoz y enlace con las administraciones de la Comunidad Autónoma de Madrid ha sido desde el primer momento la FRAVM (Federación Regional de Asociaciones Vecinales de Madrid), ha sido el encargado de mantener las comunicaciones por la vía más institucional. Sobre todo, ha tenido mucha presencia en las negociaciones con el Ayuntamiento de Madrid.

La FRAVM mantuvo una sola reunión con el Ayuntamiento de Madrid, antes ya habían atendido algunas peticiones como la creación de unas "Mesas Sociales" en los 21 distritos de la capital, algo que el Ayuntamiento de Madrid comunicó a estas Juntas de Distrito, esto sucedió el 15 de abril y solo en algunos de estos distritos (solo 4 de los 21) se crearon 
estas mesas, que tenían como objetivo generar una coordinación entre los agente sociales (Despensas Solidarias, ONG y los Servicios Sociales de cada distrito), por lo tanto el objetivo de las asociaciones y de los colectivos no llegó a cumplirse, ya que el Ayuntamiento seguía sin hacerse cargo de la situación de estas familias.

No es hasta el 12 de junio cuando el Ayuntamiento se reúne con la FRAVM ${ }^{3}$, la Red de Cuidados de Madrid tiene varias propuestas estrellas, una de ellas es la llamada "Tarjeta Monedero", una ayuda para las familias, que consiste en dar una tarjeta con dinero para que estas familias puedan comprar alimentos en los comercios de los barrios (ayudando de esta manera también al comercio local), otra de las propuestas fundamentales es la contratación de más personal para servicios sociales y la apertura de los Centros Culturales para el almacenamiento y reparto de las cestas de comida. El Ayuntamiento asumió que se iban a desarrollar todas estas medidas de manera urgente. Unas semanas después fueron aprobados por unanimidad en el Consistorio los Ilamados "Acuerdos de la Villa"4, algo que fie criticado por todos los actores sociales, ya que era un proyecto que no recogía las propuestas sociales que durante meses se habían realizado.

A día de hoy sigue existiendo una lista de espera muy amplia de familias en la Ciudad de Madrid para ser asistidos por los Servicios Sociales y la aprobación de la ayuda "Tarjeta Monedero" 5 ha llegado en el mes de agosto y solo podrán acceder a ella las familias que no reciban ninguna otra ayuda como la RMI
(Renta Mínima de Inserción), por lo que la ayuda llega a muy pocas familias.

Podemos concluir que las Instituciones madrileñas no han sabido dar una respuesta a esta situación de crisis social, que necesita de inversión pública en la parte más social. Lo que ha sucedido es que el Ayuntamiento de Madrid ha esperado a que las Despensas Solidarias cerrasen y se mantuviese el modelo de ayudas sociales anterior, basado en la caridad religiosa, dependiente de instituciones como Cáritas y Cruz Roja (externalización de la pobreza) y no ha ayudado a las asociaciones y a ese tejido a generar redes de empoderamiento y de ayuda mutua para que esta situación no se vuelva a repetir, siendo conscientes que esta pandemia no ha pasado y que lo peor desde el punto de vista social, está aún por llegar. La situación actualmente que viven los distintos municipios de Madrid respecto al número de contagios del Covid-19 se incrementa de manera desigual, siendo los barrios y municipios del sur de la Comunidad los más afectados y con cifras más altas ${ }^{6}$, que coincide además con los barrios y municipios con las rentas más bajas, con lo que podemos extraer que esta pandemia entiende de clases sociales y no afecta a todos los ciudadanos por igual.

\section{REFERENCIAS BIBLIOGRÁFICAS}

https://www.publico.es/sociedad/solidaridad-vecinal-despensas-solidarias-madrid-repiensan-futuro-frente-abandono-ayuntamiento-miles-personas-siguen-necesitandolas. html

https://www.elconfidencial.com/espa-

\footnotetext{
${ }^{3}$ https://www.europapress.es/madrid/noticia-fravm-pide-almeida-haga-cargo-cuanto-antes-ayuda-redesvecinales-atender-mas-45000-personas-20200623105205.html

${ }^{4}$ https://www.madrid.es/UnidadWeb/Coronavirus/20200702AcuerdosDELAVILLA.pdf

${ }^{5}$ https://www.eldiario.es/madrid/politica/ayuntamiento-madrid-entregara-27-000-tarjetas-credito-familias-pobres-

compra-no-reciban-ingreso-renta-minima_1_6183057.html

${ }^{6}$ https://www.elmundo.es/madrid/2020/05/13/5eb96d1efc6c83e5058b4669.html
} 
na/2020-05-18/mapa-despensas-solitarias-covid-madrid_2596671/

https://www. 20 minutos.es/noticia/2360485/0/despensas-comunitarias-madrid/autogestion-alimentaria-vecinal/red-solidaridad-popular/

https://www.bbc.com/mundo/noticias-internacional-52780033

https://aavvmadrid.org/noticias/dinamiza-tu-cuarentena-redes-vecinales-de-apoyo-ante-el-covid-19/

https://aavvmadrid.org/economia-empleo-y-consumo/238-medidas-para-la-reconstruccion-de-la-region-madrilena-post-covid-19/

https://elpais.com/espana/madrid/2020-0512/101942-madrilenos-pueblan-las-colas-delhambre-en-la-capital.html

https://aavvmadrid.org/economia-em- pleo-y-consumo/benestar-social/las-redesvecinales-distribuyen-alimentos-a-mas-de50-000-personas-en-toda-la-region/

https://transparencia.madrid.es/portales/ transparencia/es/Portada/Especial-Covid-19/Actuaciones-en-atencion-social-primaria/?vgnextfmt=default\&vgnextoid=f9a $1 \mathrm{~b}$ 5fb3e202710VgnVCM1000001d4a900aRCRD\&vgnextchannel=e8d97cbbafd71710 VgnVCM2000001f4a900aRCRD

https://www.abc.es/espana/madrid/abci-crisis-coronavirus-cada-cuatro-familias-tendra-pedir-ayudas-sociales-ayuntamiento-madrid-202005090033_noticia. html?ref=https:\%2F\%2Fwww.google.es\%2F 